\title{
Jean Rottner, le régional de la crise ? Une analyse d'activité politique en temps de coronavirus.
}

François Buton, Directeur de recherche au CNRS, Laboratoire Triangle, UMR5206, ENS Lyon. Sébastien Michon, Directeur de recherche au CNRS, Laboratoire SAGE, UMR7363, Strasbourg.

\section{Résumé}

Cet article vise à revenir sur l'activité de Jean Rottner pendant la crise du coronavirus à partir d'une analyse détaillée s de ses interventions au cœur de la crise, qui font de lui un acteur bénéficiant d'une médiatisation importante sinon extraordinaire. Pour ce faire, l'article mobilise la notion de conjoncture critique. Il défend la thèse que c'est le gestionnaire hospitalier plus que le soignant qui agit de manière ajustée dans la crise, mais aussi l'élu local qui tente de consolider sa position. L'analyse se fonde sur un dépouillement de la presse nationale et régionale ainsi que sur d'autres sources complémentaires.

Mots-clés : Rottner ; Crise sanitaire ; Région ; Gestion de crise ; Leadership local 
"C'est terrible» : le témoignage glaçant de Jean Rottner, médecin et président de la région Grand Est. Tel est le titre d'un article du Figaro repris de nombreuses fois et particulièrement commenté dans la presse. Cette prise de position de Jean Rottner, au début d'une longue séquence médiatique, marque un tournant dans le traitement médiatique et politique de la crise du coronavirus ou Covid-19 en France. De facto, cet article, paru le 15 mars 2020, soit le jour du premier tour des élections municipales, s'inscrit dans la courte séquence ouverte trois jours plus tôt par la première allocution télévisée du Président de la République, Emmanuel Macron, déclarant « la guerre » au coronavirus, et suivie le lendemain par l'annonce, au cours d'une deuxième allocution, d'une décision exceptionnelle de confinement généralisé du pays pour faire face à la vague épidémique en France. Cette décision et les mesures qu'elle entraîne modifient radicalement l'ordre institutionnel ordinaire et la division établie des secteurs d'activité au cours du printemps 2020 : report du deuxième tour des municipales, privation de libertés élémentaires durant deux mois, incitation au télétravail sauf pour les "premiers de corvée ", nationalisation du financement de 12 millions de salaires du privé, mise en suspens des injonctions budgétaires notamment pour l'hôpital public («quoi qu'il en coûte »), fermeture des frontières, mise à l'arrêt des trajets en avion et des divertissements sportifs, etc. Cet article vise à revenir sur l'activité de Jean Rottner pendant la crise du coronavirus à partir d'une analyse détaillée de ses nombreuses interventions médiatiques.

Né à Mulhouse en 1967, J. Rottner est "un gars du coin " qui présente une trajectoire professionnelle ascendante et rapide à l'hôpital de sa ville : il y est successivement médecin assistant en 1997, praticien hospitalier en 1999, chef de service des urgences SAMU-SMUR en 2003, directeur médical du pôle des urgences SAMU-SMUR en 2005. Outre son ancrage territorial, son passé d'urgentiste est un élément structurant de son identité politique, qu'il met en avant à longueur d'interviews. Si la spécialité d'urgentiste est convoquée et rappelée fréquemment, sa mobilité professionnelle à l'hôpital dans les années 2000 l'a cependant éloignée de la pratique du soin : chef de service puis directeur médical du pôle des urgences, il est passé du pôle autonome des praticiens à celui hétéronome des gestionnaires, qui en interne dirigent et managent et en externe négocient avec les responsables institutionnels et politiques. Il est aussi et surtout devenu, en 2010, un professionnel de la politique à temps complet, menant là aussi une carrière rapide et ascensionnelle. Conseiller municipal d'opposition (UDF puis UMP) à Mulhouse en 2001, sa carrière politique s'accélère suite à la victoire de Nicolas Sarkozy et de son parti aux élections de 2007. Le maire de Mulhouse, l'ex-socialiste J.-M. Bockel, devient Secrétaire d'État, chargé de la Coopération et de la Francophonie, comme «personnalité d'ouverture ». Aux municipales de 2008, Rottner devient alors le dauphin de Bockel (premier adjoint), et lui succède à la mairie en 2010 quand ce dernier accède au Sénat. Réélu maire en 2014, il cumule ensuite ce mandat à partir de décembre 2015 avec celui de vice-président de la Région Grand Est. En octobre 2017, suite à la démission de Philippe Richert, il est élu président de la Région Grand Est.

Avant la crise du Covid-19, J. Rottner est communément présenté comme un président de région

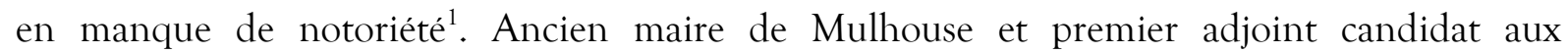
municipales en binôme avec la maire Michèle Lutz, il se trouve au début 2020 à un moment décisif de sa carrière politique. Une victoire aux municipales le renforcerait à la tête de la Région. Une défaite en revanche fragiliserait son maintien à moyen et long terme dans le champ politique local, et donc à la Région. Or, début 2020, l'issue du scrutin demeure incertaine. Le parti d'Emmanuel Macron (LREM) s'est installé dans le paysage politique local : les candidats soutenus 
par celui-ci sont arrivés en tête au premier tour de la présidentielle et des législatives 2017 à Mulhouse. Rottner est certes apparu pendant plusieurs mois comme Macron-« compatible $»^{2}$, mais une liste soutenue par LREM fait finalement face à la liste LR qu'il mène avec Michelle Lutz. Quand la crise du Covid-19 éclate à la mi-mars, cet élu de l'un des principaux foyers de contamination en France devient un "médecin en politique " : sinon l'un de ces " héros " que sont devenus les soignants applaudis tous les soirs à 20 heures par leurs concitoyen.ne.s, du moins un décideur politique régional légitime à agir et à parler en tant qu'ancien urgentiste.

La notion de conjoncture critique paraît heuristique pour penser l'activité de J. Rottner pendant la crise sanitaire (Dobry, 2009 ; Ait-Aoudia et Roger 2015 ; Gobille, 2015). Si la pandémie du coronavirus n'a pas ouvert une crise politique majeure en France, elle est par définition politique au regard des mesures prises. Les impératifs économiques ont cédé devant l'obligation politique de protéger la santé de la population - ce qui a fait débat, par exemple dans l'arbitrage entre " respect dû à "nos aînés" " et dénonciation du sacrifice économique des actifs au profit des retraités. La désectorisation a caractérisé nombre de débats ou de mobilisations. Replacer une activité dans le cadre analytique de la conjoncture critique permet de comprendre ses caractéristiques fondamentales, liées à la situation d'incertitude, et de la mesurer à l'aune de la grammaire propre aux situations de désordre : la perte d'autonomie et la porosité des frontières, donc une emprise moindre des secteurs d'activité sur les calculs et les intérêts des acteurs, ainsi qu'une situation d'interdépendance élargie, une moindre prévisibilité du monde social, un bouleversement tactique et cognitif dû l'effondrement des routines de pensée et d'action. Analyser une conjoncture critique exige de ne pas céder aux illusions de la raison héroïque et du règne des calculs, de prendre acte d'une montée en puissance de la réflexivité des acteurs dans la perception de la situation et la conduite des actions, et ouvre sur l'hypothèse de l'effectivité d'un processus de régression vers les habitus : puisque les institutions ne tiennent plus et qu'elles pèsent moins dans l'anticipation du possible, ce sont les capitaux incorporés qui sont déterminants. L'hypothèse invite dès lors à considérer que c'est bien "en tant que médecin urgentiste », spécialité qui lui donnerait une légitimité et des ressources spécifiques, que J. Rottner a agi au cours des semaines de crise, par des coups souvent ajustés à la situation. Lui-même le formule d'ailleurs ainsi : c'est parce que, "médecin à vie " ${ }^{1}$, il a jugé insupportable la pénurie en termes de matériel qu'ont dû affronter l'hôpital de Mulhouse et les professionnels de santé dans la région qu'il a agi immédiatement en conséquence, notamment en commandant des millions de masques. Néanmoins, il souligne aussi avoir agi avant tout comme président de région. De fait, à la différence d'autres médecins détenteurs d'un mandat électif, il n'intervient pas exactement en «reprenant la blouse blanche ", comme un soignant qu'il n'est plus et qu'il ne prétend plus être, mais à la fois comme président de région défendant son institution, et comme élu municipal donc gestionnaire hospitalier. Défendre son institution est décisif dès lors que la région Grand Est, issue comme d'autres d'un regroupement très récent, dispose d'une légitimité encore fragile, qu'il s'agit largement de fonder, et que, sur le plan personnel, il doit épouser un rôle dont il a hérité encore plus récemment, à la faveur d'une succession non prévue.

Cet article défend la thèse que c'est le gestionnaire hospitalier plus que le soignant qui agit de manière ajustée dans la crise. En ce sens, il invite à déconstruire deux représentations aussi enchantées que réductrices. La première repose sur l'idée d'une mobilisation générale et presque naturelle des médecins en temps de crise sanitaire, où qu'ils soient, y compris en politique. Il

\footnotetext{
${ }^{1}$ Par convention, nous indiquons les propos de Jean Rottner en italiques dans les citations pour les distinguer des propos des journalistes (sans italique).
} 
convient de rappeler à ce propos trois éléments : le champ médical est en proie à des tensions internes et à des formes de mobilisation très inégales (dans leur effectivité comme dans leur visibilité) (Pinell 2005, Pierru 2012); les situations de crise sanitaire ne supposent pas nécessairement une sollicitation des médecins au nom de leurs compétences; enfin la santé publique n'est pas l'affaire des seuls médecins et soignants, mais celle d'une diversité d'acteurs, administratifs, scientifiques et sociaux (Buton 2008). La seconde représentation s'appuie sur l'idée d'une transposabilité absolue des schèmes de perception et d'action du champ médical dans le champ politique. Cependant, la diversité des passages d'un champ à l'autre (ne serait-ce qu'en intensité et en durée ${ }^{3}$ ) ou les logiques propres au champ politique (Dulong 2010, Boelaert, Michon et Ollion 2017) invitent à remettre en question cette représentation naïve et largement produite par les médecins élus eux-mêmes (la politique consiste à diagnostiquer et soigner le corps social comme la médecine le fait pour les corps des individus, Buton et Pierru 2012).

L'analyse présentée est fondée sur un dépouillement de la presse nationale et régionale et sur d'autres sources complémentaires (dictionnaires biographiques, messages sur twitter, émissions en Facebook live, ainsi que trois entretiens avec un journaliste de la presse quotidienne régionale, un agent de la Région, et un élu local). La presse a été dépouillée de manière exhaustive sur la période comprise entre le $1^{\text {er }}$ septembre 2019 et le 11 mai 2020, jour du déconfinement national : 82 articles de la presse locale (Dernières nouvelles d'Alsace) et 145 articles issus des agences et de la presse quotidienne ou hebdomadaire nationale et régionale (cf. tableau 1 en annexe) ) $^{4}$ Cette opération permet non seulement d'appréhender les articles qui citent Rottner au cours de la période (figure 1), mais également la manière dont il est caractérisé et se présente. Un dépouillement complémentaire a porté sur les moments saillants de la trajectoire politique de J. Rottner depuis $2001^{5}$. Cette analyse est délicate faute de recul : réalisée en cours de crise, alors que la situation s'améliore à partir de mi-avril, avec une baisse régulière du nombre de cas dépistés du coronavirus, d'hospitalisations et de décès, mais que les incertitudes demeurent quant à l'apparition d'une deuxième vague à l'automne. À cette difficulté s'en ajoute une autre, bien connue des sociologues du champ médiatique (Neveu 2019) : en tant que source, la presse comporte plusieurs biais, parmi lesquels, dans le cas d'étude présent, des effets d'emballement ou de répétition (par la reprise des dépêches AFP), de compétition pour le scoop et la révélation de scandales (entre titres de la presse conservatrice, ou nationale), et de construction de la réalité sociale et politique qui lui sont propres. La mise en série d'articles n'est pas elle-même sans poser des difficultés (ne serait-ce qu'en dissociant les articles de leur contexte d'édition et de mise en page). La force de la démonstration repose ici sur le caractère systématique du dépouillement.

Adopter le cadre analytique de la conjoncture critique suppose de décrire ce qui se passe dans la crise, en se focalisant sur la dynamique de crise et l'activité tactique des acteurs (ici le seul J. Rottner, faute de place). La description adoptée distinguera trois aspects : la " réaction " quand la crise survient, l'action une fois la crise installée, et la logique générale des coups échangés au cours de la crise. J. Rottner est surpris par l'ampleur de l'épidémie, mais il est aussi bien préparé et bien placé pour en prendre conscience et réagir ; dans un premier temps, jusqu'au $1^{\text {er }}$ tour des élections municipales, son action est cependant double, entre alertes discrètes et dédramatisation publique. À partir du 15 mars, l'épidémie étant venue à lui via le cluster mulhousien, il est en première ligne, au front, «au cour de la tourmente ». Il endosse un double rôle d'organisation du front et d'anticipation de ce qui vient d'une part, et de relais d'information et de mise en alerte du niveau national d'autre part: le "général du Grand Est» (comme l'appellent ses collaborateurs) est de ce fait en position d'aborder tous les aspects de la pandémie depuis l'échelle régionale. Il intervient dès lors dans le jeu politique et médiatique national, comme le président 
de région qui, ayant un coup d'avance dans le jeu (celui de l'épidémie), entend le garder, et peut nouer ou renforcer, contre l'État central, des alliances avec ses pairs, pour l'essentiel des élus de droite dotés d'un fort capital national. Figure emblématique de la concurrence entre pouvoir national et étatique d'une part, et pouvoirs régionaux, d'autre part, mais trop peu doté pour capitaliser sur son seul nom, il demeure finalement (à la date du 11 mai 2020) le « régional de la crise ", dont les profits politiques sont avant tout locaux et régionaux.

Figure 1 : nombre d'articles qui citent Jean Rottner selon la semaine et le type de média (entre le 6 janvier et le 11 mai 2020) 6

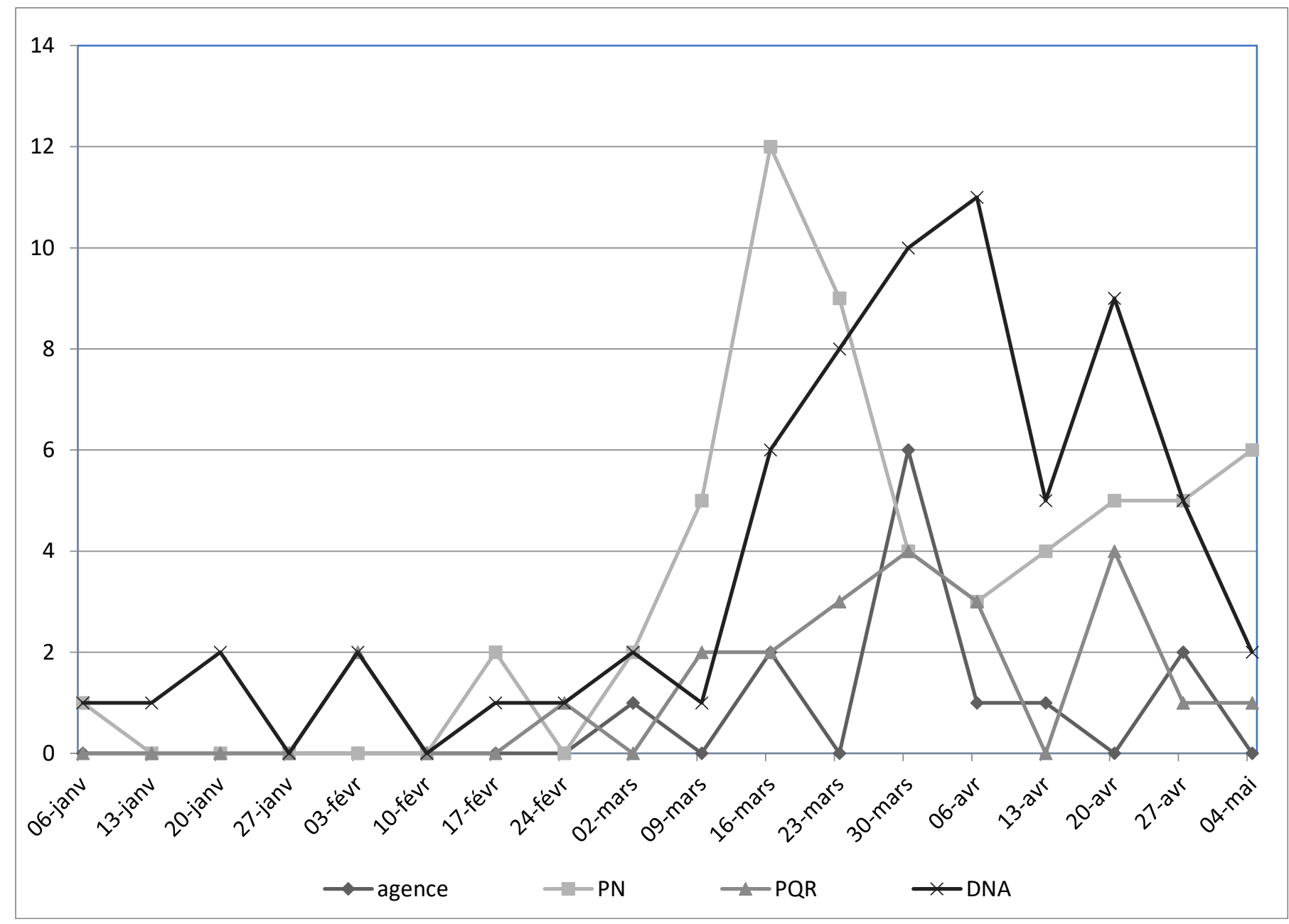

Champ : l'ensemble des articles qui citent J. Rottner entre le 6 janvier et le 11 mai. Les articles $(\mathrm{N}=161$ au total) sont différenciés selon 4 sources : agence $(\mathrm{N}=13)$, presse nationale $(\mathrm{PN})(\mathrm{N}=58)$, Dernières nouvelles d'Alsace (DNA) $(\mathrm{N}=67)$, Presses quotidiennes régionales en dehors des DNA $(\mathrm{N}=23)$

Commentaire : le graphique atteste l'augmentation très forte de la couverture médiatique de J. Rottner de manière générale sur la période de la crise (mi mars à mi mai), qui culmine avec l'arrivée de l'épidémie à Mulhouse (pour la presse nationale) et avec la guerre des masques (pour les agences, les DNA, et la presse quotidienne régionale).

\section{Bien préparé pour faire face à la crise}


Comme tous les responsables politiques, J. Rottner est surpris par l'arrivée du coronavirus en France ; ce qui le distingue des autres, c'est d'abord que l'épidémie vient à lui, dans sa ville, en raison, dit-on (en mai 2020), du rassemblement de l'Église protestante évangéliste Porte Ouverte Chrétienne $^{7}$. La coïncidence est trop frappante pour ne pas être rappelée : le Président Macron se rend à Mulhouse le 18 février pour "dénoncer le séparatisme musulman " au cours de la semaine même où, à quelques centaines de mètres du lieu de son intervention, le rassemblement de milliers d'évangélistes (du 17 au 21) est en train de créer le bouillon de culture à l'origine de la propagation du virus dans l'agglomération, en Alsace, et (très largement) dans le reste du pays. L'intervention présidentielle s'inscrit dans un contexte politique plus général marqué par le projet gouvernemental de loi sur les retraites, fortement contesté depuis décembre, mais adopté sans vote à l'Assemblée début mars, et par la campagne des élections municipales prévues pour les 15 et 22 mars. Interrogé sur le discours présidentiel du 18 février, J. Rottner défend son action d'édile mulhousien opposé au fondamentalisme salafiste, mais favorable au dialogue avec la communauté musulmane ${ }^{9}$. En ce début d'année, le président de région est évoqué assez rarement dans la presse nationale et régionale ${ }^{10}$, par exemple pour sa politique d'ouverture du marché du transfert ferroviaire, la cyberattaque contre l'administration régionale intervenue le 14 février, ou sa défense du biocarburant lors du Salon de l'agriculture à Paris ${ }^{11}$. Début février, un portrait particulièrement flatteur est dressé par «son» opposante socialiste à la Région, Pernelle Richardot: républicain, exigeant, imaginatif, fidèle politiquement, autoritaire, mais sachant consulter, c'est un "médecin dans le public " et un "maire qui aime les gens " ${ }^{12}$. Après avoir passé une journée avec lui, L'Union ne peut décrire la vie du président Rottner autrement que comme une " course contre le temps ». Il s'y dépeint comme non destiné à occuper son poste, devant encore se faire connaître, attaché au «terrain ", au travail d'équipe, pétri d'humilité, pragmatique, et qui n'envisage pas son avenir ailleurs que dans sa région : "Je n'ai reçu aucune sollicitation pour intégrer le gouvernement depuis 2017, et même à l'époque de Nicolas Sarkozy, n'ayant jamais rien fait pour (...) Solliciter un nouveau mandat (...) ? Il est trop tôt pour se prononcer, je vais déjà aller au bout de celui-ci. Je donne le maximum de moi-même, j'essaye d'incarner le Grand Est " ${ }^{13}$. Dans la presse locale en revanche, c'est surtout le candidat aux élections municipales qui est évoqué. En ce début d'année, le calendrier de J. Rottner est largement indexé sur l'échéance électorale.

Une dizaine de jours après le rassemblement religieux, Mulhouse et ses environs sont devenus " le " foyer majeur de l'épidémie en France. Alertés, le préfet et le directeur de l' Agence régionale de santé (ARS) convoquent une réunion le 2 mars (dont J. Rottner rend compte sur twitter dès le lendemain), puis provoquent " une réunion de crise " le 6 mars. Le même jour, J. Rottner se veut rassurant : "nous relevons les manches, et nous souhaitons nous en sortir. La crise va être longue, cela va être difficile, elle risque d'affoler la population. Je fais donc un appel au calme et à la raison pour maîtriser l'épidémie " ${ }^{14}$. Le 8 mars, alors que l'hôpital de Mulhouse passe en plan blanc (dispositif de mobilisation maximale face à une situation sanitaire exceptionnelle), il se félicite des mesures de fermeture de locaux décidés par l'État et la mairie ${ }^{15}$ et entend "rassurer et protéger et les électeurs et le personnel mobilisé à l'occasion de ces élections » à une semaine de l'échéance électorale ${ }^{16}$. C'est avant tout comme maire-adjoint, à ce titre président du Groupement régional hospitalier MulhouseSud Alsace, qu'il s'exprime et vante la mobilisation des personnels hospitaliers ou la coopération avec la médecine de ville. "La situation est maîtrisée " ${ }^{17}$. Le 12 mars, jour de la première intervention martiale et alarmiste du président Macron, le ton est aussi à la mobilisation chez le président du Grand Est, mais avec distance : même si la région est « sur le pied de guerre » face à l'épidémie, il refuse de " tomber dans la psychose » et décrit lui-même « avec ironie » le Grand Est comme une "région pilote ${ }^{18}$. "L'hôpital absorbe la première salve de manière correcte ", le principal point noir étant l'épuisement des professionnels de santé. À deux jours de l'élection, il soutient 
le maintien du scrutin décidé par l'Élysée, «instant démocratique qui doit se réaliser, la majorité de notre territoire [n'étant] pas viralisé actuellement ». Mais il se place aussi en poste avancé de la lutte contre l'épidémie : "Depuis Mulhouse, nous savons donc ce qu'il va se passer à l'échelle du pays, d'où l'importance des gestes et mesures de prévention " ${ }^{19}$. La veille du 15 mars, c'est surtout "préparer la reprise " économique qui intéresse son entourage ${ }^{20}$.

Finalement, au cours des jours qui précèdent l'élection, J. Rottner tient le discours plutôt rassurant d'un élu pas mobilisé à plein temps par la lutte contre l'épidémie, car engagé dans la campagne des municipales. Pourtant, en off, il a alerté dès le 5 mars le président de la République par un texto dont il révèle plus tard le contenu ${ }^{21}$ : "Sans être alarmiste, la situation haut-rhinoise devient réellement endémique. J'ai passé quatre heures à la régulation du SAMU hier soir et j'ai pu apprécier la diffusion du virus. Rester au stade 2 alors que nous nous comportons petit à petit comme si nous étions au stade 3 ne me semble pas réaliste. Nous faisons tout ce que nous pouvons, mais la parole publique risque d'être dépassée par les faits ». C'est seulement le dimanche même du premier tour que le cri d'alarme devient public, que tout bascule : "c'est terrible», déclare-t-il au Figaro ${ }^{22}$. Désormais, face à "l'ouragan", au "tsunami", au "rouleau compresseur", à la "bombe à fragmentation " qu'est l'épidémie à Mulhouse ${ }^{23}$, J. Rottner fait feu de tout bois.

Surpris par le nombre des cas, dont il prend sans doute conscience au cours de la semaine du 8 au 15, J. Rottner est à la fois bien placé pour comprendre ce qui se passe, et en position de se donner le juste rôle dans la bataille en première ligne contre le coronavirus. Président du conseil d'administration de l'hôpital, il est au courant des grandes difficultés rencontrées par les services, et notamment par les urgences de Mulhouse depuis plusieurs mois (départs non remplacés de médecins, grève des internes), son ancien service, au chevet duquel il s'est rendu à l'automne $2019^{24}$. Comme il le reconnaît bien plus tard, il n'est certes plus en capacité d'exercer comme médecin aux urgences, mais il peut recevoir les appels du 15, bref se placer en "soutien " aux soignants ${ }^{25}$ : «je n'étais pas au front comme d'autres peuvent le faire. (...) Quand on vient donner un coup de main comme ça, il faut juste rester à sa place " ${ }^{26}$. Sa connaissance du milieu hospitalier, son expérience des plans blancs, ses vacations téléphoniques au 15 ou ses échanges avec "ses " chefs de service (qui sont à bout ${ }^{27}$ ) sont décisifs pour l'amener à endosser son rôle, comme il le raconte une fois le pic de l'épidémie passé : "mon expérience de médecin a sûrement joué et m'a obligé, comme élu, à avoir à la fois un devoir d'alerte et de protection. Quand j'ai alerté il y a quatre semaines sur la situation terrible que nous vivions, c'était le médecin qui parlait. Quarante-huit heures plus tôt, je régulais aux urgences et voyais que quelque chose de grave se passait. J'étais en deçà de la vérité. (...) Si on ne bouge pas à ce moment-là, on n'assume pas son rôle. Médical ou politique? Peu importe comment on l'appelle. Il fallait que j'aille au-delà de mes compétences propres, que je prenne des risques... ${ }^{28}$. Discours classique du médecin en politique qui souligne la continuité entre les deux activités sous l'angle de l'alerte par le diagnostic, et de la protection par la prévention et le soin, comme le résume Le Monde dans le premier portrait qu'il lui consacre : "politique et urgentiste, c'est le combo gagnant du moment. "Général du Grand Est" dans la "guerre" menée contre l'épidémie, ose un de ses proches collaborateurs, Jean Rottner est devenu incontournable " ${ }^{29}$.

\section{En première ligne : facilitateur et lanceur d'alerte}

Pour le général-président, le rôle se concrétise donc sous une double forme, courroie de transmission entre diverses autorités ou " facilitateur " ${ }^{30}$, et lanceur d'alerte. D’une part, J. Rottner 
facilite la collaboration avec l'État, le monde économique et les partenaires étrangers. Il échange régulièrement par textos avec les sommets de l'exécutif et se félicite dès le 16 mars de l'arrivée prochaine d'un hôpital militaire de campagne sur le parking de l'hôpital de Mulhouse (où Macron revient pour une allocution officielle le 25 mars) ${ }^{31}$. Au monde économique, il déclare qu'il a besoin de lui pour réunir des masques FFP2 pour les soignants. Quant aux partenaires allemands d'abord, puis suisses et luxembourgeois, il les sollicite pour recevoir des malades en réanimation et désengorger le service mulhousien. Mais d'autre part il se définit aussi (et est très vite reconnu ${ }^{32}$ ) comme un lanceur d'alerte, pour son texto au président bien sûr, mais aussi par ses sorties médiatiques, où, de plus en plus souvent présenté par son ancienne spécialité d'urgentiste, il déploie deux arguments principaux, établis dès la semaine du 16 mars. Le premier, hospitalo-centré, dénonce avec force la pénurie des moyens de l'hôpital, notamment en masques, mais aussi en blouses, en respirateurs, etc., pénurie intolérable alors que les services sont saturés et les soignants épuisés ("vrais héros, face à la mort, à la peur, au stress »), auxquels très vite il associe tous ceux qui sont "à l'avant ", pompiers, caissières, forces de l'ordre, etc. ${ }^{33}$. Le second, d'ordre préventif, critique durement les Français qui ne respectent pas le confinement et les "gestes barrières" ("ce n'est pas faire le malin, c'est faire le con " ${ }^{34}$ ), et les appellent fermement à une prise de conscience. Ces discours de mise en scandale de la pénurie d'une part, des comportements d'autre part convergent autour de la question des masques : dès le 21 mars, alors que le discours gouvernemental est empêtré dans des contradictions sur leur disponibilité, leur utilité ou encore leurs difficultés d'usage, il appelle à "arrêter maintenant avec cette doctrine qui dit que le masque ne sert à rien " ${ }^{35}$. Les masques ne devraient pas manquer, ils sont utiles, et tout le monde doit en porter : la position de J. Rottner détonne dans le paysage politique par sa fermeté, sa continuité, et sa précocité.

Loin de se contenter de dénoncer la pénurie, J. Rottner engage une politique d'approvisionnement en masques en faveur des soignants et de ceux de l'avant : il réquisitionne les stocks disponibles dans les lycées, sollicite les dons des entreprises, et surtout effectue, le premier, une commande à des producteurs chinois. Présentée sur France Info le 21 mars, la commande des 5 millions de masques fait sensation jusque dans L'Humanité ; ils sont livrés le $1^{\text {er }}$ avril. Fort de ce titre de précurseur, il s'engage publiquement dans un sujet à très forte valeur médiatique, élevé au rang de "guerre ", entre le 21 mars et 12 avril ${ }^{36}$. Sans entrer dans les détails, il s'avère que la concurrence pour les masques existe à un double niveau : international et national ${ }^{37}$. À l'échelon international, J. Rottner et Renaud Muselier, président de la Région Sud, déclarent indignés sur plusieurs antennes radiophoniques les $1^{\text {er }}$ et 2 avril que les Américains "sortent le cash" pour acheter des masques avec des "méthodes de cow-boy " "sur le tarmac" des aéroports chinois, relayant une information que leur a confié Valérie Pécresse, président de région Île-de-France, furieuse d'avoir perdu une commande ${ }^{38}$. Après un démenti des États-Unis, J. Rottner se rétracte en dénonçant une opération de "désinformation " ${ }^{39}$. À l'échelon national, c'est la présidente de région Bourgogne-Franche-Comté qui se plaint le 5 avril que le Grand Est aurait " détourné » des masques qui lui étaient destinés. Il s'avère en fait que c'est l'État, via l'ARS Grand Est, qui a ordonné la saisie des masques ${ }^{40}$, mais d'autres cas ayant eu lieu, au détriment notamment de la région $\mathrm{Sud}^{41}$, J. Rottner saisit l'occasion pour émettre une première critique de l'action de l'État et se rapprocher des autres présidents de région. Les deux mini-scandales lui permettent de mettre en valeur son sens de l'anticipation et de l'organisation : dans un contexte de compétition acharnée, lui a su installer "une petite cellule au niveau de la région qui travaille d'arrache-pied pour, avec les commanditaires, pouvoir gagner ces marchés ${ }^{42}$, et le faire assez tôt, avant que les Américains n'interviennent, le jour même où un décret l'a rendu possible. D'après Le Point, "l'impréparation des Français est donc la vraie cause de leurs difficultés. Si le Grand Est 
s'en est mieux tiré que les autres, c'est qu'il a pu mobiliser le premier ses trois importateurs, dont le principal est un français qui fournissait déjà des centres hospitaliers universitaires " ${ }^{43}$.

\section{Incarner et défendre la région, agir avec et contre l'État}

En situation de crise et d'incertitude, J. Rottner a pris un coup d'avance sur la question des masques, et a su le faire savoir ; il s'est aussi montré constant dans sa recommandation de l'usage généralisé des masques. Il s'agit là d'un avantage certain dans le champ politique, sur un sujet de plus en plus construit médiatiquement et politiquement comme un "scandale d'État " (leur inutilité ayant été proclamée par les responsables politiques et administratifs en mars en raison de... leur pénurie $\left.{ }^{44}\right)$. Au cours du mois d'avril, il mobilise le registre du coup d'avance en s'attaquant au marché des tests sérologiques ou en se plaçant en prescripteur pour la phase de déconfinement prévue le 11 mai ; il bénéficie alors d'une reconnaissance élargie au niveau national, d'un capital politique nouveau, au moins médiatiquement, mais dans les habits déjà bien connus du responsable de collectivité locale en butte à l'État, comme incarnation (mesurée) de la figure (familière) du girondin en politique.

Au cœur de la vague sanitaire en Alsace, de mi-mars à mi-avril, J. Rottner, " politique de l'urgence à la tête de la région Grand Est ", est sollicité pour de longs entretiens dans la presse nationale (Le Figaro est sa principale chambre d'écho, Le Point le cite souvent, Le Monde plus marginalement, cf. tableau 1). Dans Le Point du 17 avril, le président « en première ligne depuis l'arrivée de la vague qui a déferlé sur Mulhouse ", "n'élude aucun sujet ", et - on y revient -, prend de la hauteur : "partisan d'une "union nationale", il prône un "nouveau modèle économique" et en appelle à un "pacte social" pour répondre à la colère qu'il sent "monter" passée la sidération ". Il présente aussi longuement la société d'économie mixte Dynamise, créée pour financer l'achat de 3 millions de tests sérologiques : "Avec la banque des territoires et le groupe Crédit Mutuel, on a mis en place la boîte qui va nous permettre d'anticiper, de ne pas entrer dans la même guerre que celle que nous avons vécue pour les masques. Pour une fois, ayons un coup d'avance". J. Rottner n'entend pas jouer aux "francs tireurs " dans une "course à l'échalote " où il s'agirait d'être "le plus rapide ", mais simplement " accélérer le mouvement " de la reprise, qui sera difficile dans la région la plus touchée de France. Ce projet est largement évoqué dans la presse régionale et nationale entre le 12 avril et le 22 avril. D'autres présidents (comme Alain Rousset en Nouvelle Aquitaine) y réfléchissent aussi, dans un contexte de "guerre industrielle" dans laquelle une société strasbourgeoise est bien placée. Cependant il est aussi décrit comme ambivalent ("anticipation ou coup de com' "?) et d'autant plus mis en cause que la fiabilité des tests sérologiques est loin d'être garantie ${ }^{45}$. Et il fait lui aussi l'objet d'une concurrence avec l'État, bataille de mots, mais aussi divergence de positions : suivant un avis de la Haute Autorité de Santé (en date du 16 avril), l'ARS Grand Est renonce le 23 avril à l'usage des tests sérologiques dans les EHPAD ${ }^{46}$.

Ayant, selon Le Monde, " choisi de se placer aux avant-postes, quitte à multiplier les tensions avec une agence régionale de santé (ARS) qu'il critique volontiers ", J. Rottner se félicite que «depuis le début, la région donne le "la" au niveau des actions menées " ${ }^{47}$. Or, l'antagonisme entre le président de région et l'État est mis en scène alors même que les plus hauts dirigeants sont amenés presque par nécessité à fouler le sol alsacien. Le président Macron prononce le 25 mars une allocution officielle depuis le parking de l'hôpital de Mulhouse où a été installé par l'armée un hôpital de campagne ; J. Rottner n’a pas pu participer à la réunion, car « en médecin », il s'est confiné la veille après l'apparition de symptômes finalement non liés à l'infection par le coronavirus ${ }^{48}$. Un mois 
plus tard, c'est le Premier ministre Philippe qui rend une visite surprise à Strasbourg pour discuter avec les élus alsaciens à quelques jours de la présentation de la "stratégie nationale " pour le déconfinement fixé par le Président au $11 \mathrm{mai}^{49}$. Présent, J. Rottner plaide avec ses collègues pour un déconfinement "différencié " par région, et reprend, sans le dire explicitement, certains des pré-requis définis par le Conseil scientifique placé auprès du Président, comme la mise en place d'indicateurs nationaux pour le suivi de l'épidémie - que le Premier ministre validera effectivement quelques jours plus $\operatorname{tard}^{50}$. Sa position, là encore en avance, politiquement au moins, sur l'enjeu du déconfinement, devenu un sujet majeur en France entre la mi-avril et la mimai, se traduit avec éclat par la parution, dans Le Figaro du 19 avril, d'une tribune cosignée, en tant que président LR de Grand Est, mais aussi « médecin urgentiste de profession », avec Philippe Juvin, pour sa part chef de service des urgences de l'hôpital européen Georges-Pompidou à Paris, mais aussi maire LR de La Garenne-Colombes (Hauts-de-Seine), et expert très fortement sollicité dans les médias notamment télévisuels depuis le début de la crise - " roi des directs ", il fait partie des cinq " médecins incontournables " dans la crise identifiés par L'Express ${ }^{51}$. Les deux médecins élus détaillent sept conditions " médicales " pour réussir le déconfinement, ou au moins empêcher une nouvelle vague épidémique. De la nomination d'un décideur ou "pilote unique dans l'avion " à la création d'une "réserve stratégique de lits de réanimation » en passant par le port des masques ou les pratiques d'isolement des cas positifs, ces conditions déclinent un plan stratégique alternatif à celui présenté par le gouvernement, même s'il s'en rapproche beaucoup. Dans la forme, la tribune signe une prise de position qui consacre le capital politique spécifique que Rottner a accumulé.

Ce capital n'est pas seulement médiatique. Membre du parti Les Républicains mais issu du centre politique, J. Rottner est promu par des acteurs importants de la fraction conservatrice du champ politique. L'eurodéputé LR Arnaud Danjean le distingue parmi les « élus locaux extrêmement performants, reconnus, qui se battent tous les jours pour trouver des solutions sur le terrain $»^{52}$. Le sénateur et ancien ministre Gérard Longuet le cite en " exemple parfait " de la mobilisation du secteur médical privé (ville et hospitalisation) ${ }^{53}$. Avant eux, l'ancien président Nicolas Sarkozy l'avait désigné comme l'un des rares, parmi ses proches (avec... Philippe Juvin ou Jean Castex, "Monsieur Déconfinement») à le tenir informé de la situation sanitaire ${ }^{54}$. La reconnaissance dépasse le champ politique proprement dit : dans une interview fleuve accordée au Figaro sur la pandémie comme "retour, bienvenu, du tragique », le politologue Pascal Perrineau, lui-même consacré médiatiquement, avait souligné « les écarts de fonctionnement d'une région à l'autre " et vanté l'" activisme ", le "sens de l'anticipation (...) et de l'initiative remarquables " de J. Rottner ( il est vrai médecin-urgentiste de profession »), pour mieux regretter que les pouvoirs des régions restent "modestes $»^{55}$. On le voit, le capital politique acquis lors de la crise est très spécifique : c'est en tant que président de région que Rottner est reconnu, ce qui tient aussi à ses prises de position contre l'État.

S'il est délicat de dater le début d'un discours critique contre l'État chez J. Rottner, tant il s'agit d'un lieu commun chez les présidents de région, on peut considérer que la montée en épingle intervient mi-avril, autrement dit après le pic de la crise, et surtout dans Le Point, donc dans la foulée de la couverture par ce journal de la «guerre des masques " et plus généralement de sa ligne éditoriale anti-étatique ${ }^{56}$. Dans un premier bilan du rôle des présidents de région dans la crise $^{57}$, et notamment de Rottner, érigé en " pionnier ", L’Express souligne ainsi avec admiration qu'en multipliant les initiatives, les élus locaux ont su « [monter] au front pour pallier les carences de l'État ${ }^{58}$. Auparavant, J. Rottner n'aborde guère la question de la contribution de l'État, absente par exemple des deux entretiens réalisés fin mars avec les journalistes du Figaro et du 
Monde comme de la première tribune qu'il co-signe avec 13 autres élus locaux de droite. Intitulée "Face à l'épidémie, organisons la solidarité entre nos régions et à l'échelle européenne ", et publiée dans Le Figaro du 29 mars, celle-ci réunit notamment des présidents de région (V. Pécresse et J. Rottner), mais aussi entre autres les présidents des deux départements alsaciens (Frédéric Bierry et Brigitte Klinkert), le député de Mulhouse Olivier Becht ou l'ancienne maire de Strasbourg Fabienne Keller. Dans le cadre général d'une solidarité entre régions d'Europe et en réponse à un appel de l'incontournable Philippe Juvin, l'idée consiste à faire pour l'Ile-de-France ce qui a été réalisé dans le Grand Est à savoir un transfert massif de patients en réanimation ; on n'y lit aucune critique de l'État et même, au contraire, un appui aux déclarations du Président de la République sur la "guerre au virus ".

Dans les faits, les prises de position de J. Rottner s'inscrivent principalement dans la dénonciation très médiatique des «boulets bureaucratiques " que seraient les ARS, défaillantes dans la gestion de l'approvisionnement en masques, capables de produire des normes, mais pas de gérer une crise, administrations "puissantes", mais "obèses", éloignées en bref du terrain ${ }^{59}$. Dans l'entretien, très politique, qu'il accorde au Point le 17 avril, il demande avec prudence « davantage de décentralisation " en faveur des collectivités locales jugées "plus agiles et plus réactives ", sans pour autant endosser "l'opposition entre jacobins et girondins ». Il se fait plus virulent et concède "pester " contre les ARS, "État dans l'État ", à qui on demande depuis des années de "gérer des budgets et fermer des services", et qui se seraient avérées incapables de "devenir des spécialistes de la gestion de la crise ": "ça ne marche pas, ça ne peut pas marcher, et, d'ailleurs, je ne leur en veux pas " ${ }^{60}$. La critique se déploie à nouveau fin avril, alors que les conditions pratiques du déconfinement sont débattues. J. Rottner soutient certes les mesures annoncées par le Premier ministre le 28 avril, notamment le principe d'une différenciation régionale qu'il avait appelé de ses vœux ${ }^{61}$, mais il monte en critique à l'égard de la gestion de l'épidémie en région au cours d'une table-ronde sur "Les élus à l'action dans les foyers épidémiques de COVID-19", organisée le 30 avril par la délégation aux collectivités territoriales du Sénat. Il dénonce fermement la gestion bicéphale de l'épidémie par le préfet de région et l'ARS et "l'incapacité des autorités sanitaires nationales et régionales à réagir rapidement, en termes de matériel notamment ", et réclame une réforme "en profondeur de notre administration de santé ", qu'il faut "remettre sous l'autorité du préfet en période de crise pour que l'articulation avec les collectivités puisse se faire " ${ }^{62}$. Autrement dit, il s'autorise une critique des politiques de santé qui ne dépasse pas le niveau régional de sa mise en œuvre. Dans la construction d'une adversité entre régions et État, il s'en tient finalement à un éloge des capacités d'action régionales et une critique des seuls échelons déconcentrés de l'action étatique.

À l'issue de la phase de déconfinement (11 mai 2020), le politiste dispose d'une abondance de déclarations, de citations et d'entretiens de J. Rottner, qui frappent par leur cohérence. L'analyse de son activité dans le contexte de conjoncture critique créé par la pandémie du coronavirus montre qu'il a agi en gestionnaire hospitalier plutôt qu'en soignant. Si le président de la région Grand Est a acquis une notoriété médiatique nationale, son capital politique national reste largement à établir, en grande partie parce que l'intéressé semble préférer se cantonner au rôle de leader local et régional. Cette modestie tient sans doute davantage à la vulnérabilité de son double leadership au moment où l'épidémie surgit qu'à un manque "d'ambitions » du " petit urgentiste de province " et gestionnaire hospitalier, même si le sentiment de moindre légitimité par rapport à ses pairs présidents de régions et/ou technocrates " prédisposés » à la politique n'est pas à écarter. De fait, quand avec le déconfinement, Les Républicains entendent incarner l'alternative à Macron, Rottner n'est plus qu'un président de région parmi d'autres, dont l'action 
est saluée comme pionnière, mais dont le poids politique demeure en deçà d'autres présidents eux aussi présentés comme exemplaires (Pécresse, Bertrand, Wauquiez). Il est d'ailleurs frappant que dans sa contestation de l'action des échelons régionaux de l'administration d'État (préfet et ARS), J. Rottner porte la critique sur les savoir-faire administratifs, trop budgétaires et pas adaptés à la gestion de crise, plutôt que sur les conséquences de la reconfiguration des régions, dont les ARS ont, elles aussi, subi les frais, ou sur la politique nationale de sécurité sanitaire face à la crise. En ce sens, la portée critique ne vise pas les cadres nationaux de la pratique politique, mais leur exercice au niveau régional.

Dans la production journalistique décrivant l'action de J. Rottner en temps de coronavirus, un document hors corpus conforte l'hypothèse du "régional de la crise ". Le 16 mai 2020, La Croix hebdo lui consacre un long portrait illustré qui résume parfaitement le rôle qu'il s'est, et qu'on lui a, donné. Un rôle fait de modestie devant le destin politique en raison de sa trajectoire de médecin : "toute ma carrière politique est faite de moments qui se sont présentés et que je n'avais pas prévus (...). Quand vous êtes médecin, a fortiori urgentiste, vous ne vous prédisposez pas à la politique. Ce sont des portes qui s'ouvrent, on en franchit le seuil, voilà "; "mon expérience de médecin m'a appris le travail en équipe, la prise de décision rapide, mais aussi l'humanité, et l'humilité face à l'échec et à la mort ». Une modestie liée aux mandats même d'élu local, qui doit agir, mais en situation de proximité : " J'ai été maire et j'essaie d'adopter la même logique de proximité à la tête de la région "; "Comme élu local, je considère que j'ai un devoir de protection et un devoir d'action. C'est ce que j'ai essayé de décliner depuis le début de cette crise ». Finalement, c'est un pragmatisme de bon aloi que revendique J. Rottner, depuis la position qu'il occupe : "Essayons déjà de résoudre cette crise énorme. Elle nécessite que chacun reste à sa place».

\section{Références}

Ait-Aoudia, Myriam et Roger, Antoine (dir.) (2015), La logique du désordre: Relire la sociologie de Michel Dobry. Paris, Presses de Sciences Po

Boelaert, Julien, Michon, Sébastien et Ollion, Étienne (2017), Métier : député. Enquête sur la professionnalisation de la politique en France, Paris, Raisons d'agir.

Buton, François (2008), «Sous l'emprise des risques sanitaires : les métamorphoses de la santé publique ", Savoir/Agir, n5, p. 75-82.

Buton, François et Pierru, Frédéric (2012), "Les dépolitisations de la santé ", Les Tribunes de la santé, $\mathrm{n}^{\circ} 34$, p. $51-70$

Dobry, Michel (2009), Sociologie des crises politiques: La dynamique des mobilisations multisectorielles. Paris, Presses de Sciences Po.

Dulong, Delphine (2010), La construction du champ politique en France, Rennes, Presses universitaires de Rennes.

Gobille, Boris (2015), «De l'étiologie à l'historicité des crises: Sociologie des crises politiques et sociohistoire du temps court ", in Aït-Aoudia ; Myriam et Roger, Antoine (éd.), La logique du désordre: Relire la sociologie de Michel Dobry, Paris, Presses de Sciences Po, p. 153-176.

Neveu, Érik (2019), Sociologie du journalisme, Paris, La Découverte. 
Pinell, Patrice (2005), "Champ médical et processus de spécialisation ", Actes de la recherche en sciences sociales, $\mathrm{n}^{\circ} 156-157$, p. 4-36.

Pierru, Frédéric (2012), «Le mandarin, le gestionnaire et le consultant. Le tournant néolibéral de la politique hospitalière ", Actes de la recherche en sciences sociales, n¹94, p. 32-51.

\section{Annexe}

Tableau 1 : Source des articles consultés parus entre le $1^{\text {er }}$ septembre 2019 et le 11 mai 2020 qui évoquent ou citent Jean Rottner

\begin{tabular}{|c|c|c|c|c|}
\hline Catégorie & Sous-catégorie & Titre & Journaux recodés & Rottner cité \\
\hline \multirow[t]{2}{*}{ Agence } & Agence & AFP & 15 & 10 \\
\hline & & Reuters & 3 & 3 \\
\hline Site & Site & BFM.info & 1 & 1 \\
\hline \multirow[t]{19}{*}{ Presse nationale (PN) } & $\begin{array}{l}\text { Presse magazine } \\
\text { nationale }\end{array}$ & Le Point & 8 & 6 \\
\hline & & La Gazette des communes & 4 & 2 \\
\hline & & Le Quotidien du Médecin & 4 & 1 \\
\hline & & L'Obs & 3 & 3 \\
\hline & & L'Express & 3 & 2 \\
\hline & & Bulletin quotidien & 1 & 1 \\
\hline & & Le Courrier des maires & 1 & 1 \\
\hline & & Paris Match & 1 & 1 \\
\hline & & Valeurs actuelles & 1 & 1 \\
\hline & & Courrier international & 1 & 0 \\
\hline & $\begin{array}{l}\text { Presse quotidienne } \\
\text { nationale }\end{array}$ & Le Figaro & 24 & 11 \\
\hline & & Le Monde & 12 & 9 \\
\hline & & Les Echos & 7 & 6 \\
\hline & & Aujourd'hui & 6 & 6 \\
\hline & & La Tribune & 5 & 2 \\
\hline & & Libération & 3 & 2 \\
\hline & & L'humanité & 2 & 2 \\
\hline & & La Croix & 2 & 1 \\
\hline & & 20 minutes & 1 & 1 \\
\hline \multirow[t]{3}{*}{$\begin{array}{l}\text { Presse quotidienne } \\
\text { régionale (PQR) }\end{array}$} & PQR hors Alsace & L'Est Républicain & 21 & 14 \\
\hline & & L'Union & 6 & 4 \\
\hline & & Sud-Ouest & 4 & 3 \\
\hline
\end{tabular}




\begin{tabular}{|l|l|l|c|c|} 
& & Journal du Grand Paris & 1 & 1 \\
\hline & & Midi Libre & 1 & 1 \\
\hline & & Ouest France & 1 & 1 \\
\hline & & Paris-Normandie & 1 & 1 \\
\hline & & Le Bien Public & 1 & 0 \\
\hline & & Le Télégramme & 1 & 0 \\
\hline & & Dernières nouvelles d'Alsace & 82 & 44 \\
\hline & & Total & $\mathbf{2 2 7}$ & $\mathbf{1 4 1}$ \\
\hline
\end{tabular}

1 «Les Français très attachés à leur région », Le Figaro 13 décembre 2019.

${ }^{2}$ Le Figaro 13 mars 2018.

${ }^{3}$ Sur ce point, on renvoie aux travaux du colloque « Professionnels en "déplacement". Ce que passer les frontières professionnelles veut dire » organisé à la MSH-LSE, Lyon, 14 et 15 novembre 2019.

${ }^{4}$ Le dépouillement exhaustif de la presse non locale a été réalisé sur Europresse, qui regroupe 156 sources pour la France.

${ }^{5}$ Soit les élections municipales de 2001, 2008, 2014 et 2020, législatives de 2002, l'élection en tant que maire de Mulhouse en 2010, l'élection à la présidence de la région Grand Est en 2017, ainsi que l'entrée au gouvernement de Jean-Marie Bockel en 2007.

${ }^{6} \mathrm{PQR}$ sans DNA.

${ }^{7}$ Les autres premiers cas groupés en février, dans l'Oise ou en Savoie, sont davantage circonscrits, si l'on en croit la mesure de la diffusion des hospitalisations.

${ }^{8}$ Le Figaro 18 février.

${ }^{9}$ Le Figaro 18 février.

${ }^{10}$ La base Europresse permet d'identifier 145 articles de presse citant le nom de Rottner au moins une fois entre le $1^{\text {er }}$ septembre 2019 et le 10 mai 2020. Mais 19 articles seulement paraissent entre septembre et février (soit 6 mois), contre 49 en mars, 64 en avril. 7 articles sont comptabilisés pour le seul 30 avril, soit autant que janvier et février réunis.

${ }^{11}$ Tribune 10 janvier, Gazette des communes 21 février, L’Est Républicain 26 février.

${ }^{12}$ L'Union 4 février.

${ }^{13}$ L'Union 5 février.

${ }^{14}$ Aujourd'hui 6 mars (site web) et 7 mars (papier).

${ }^{15}$ Le Figaro 8 mars.

${ }^{16}$ AFP 8 mars.

${ }^{17}$ Le Figaro 8 mars, Quotidien du médecin 10 mars.

${ }^{18}$ Le Monde 12 mars.

${ }^{19}$ L'Union 13 mars.

${ }^{20}$ L'Est Républicain 14 mars.

${ }^{21}$ Par exemple, Figaro 23 mars.

${ }^{22}$ Figaro 15 mars.

${ }^{23}$ Tous ces termes seront employés au fil des semaines.

${ }^{24} \mathrm{Au}$ sein duquel il a fait « son retour », les sources divergent sur le sujet (DNA 21 septembre, AFP 15 octobre, Quotidien du médecin 16 octobre, L'union 17 octobre, mais aussi Echos 19 mars). L'intérim assuré en « mouillant la blouse » en septembre octobre est dénoncé par certains comme « un coup de com' » alors que Jean-Marie Bockel se dit certain de sa sincérité « dans son engagement d'urgentiste » (Le Monde 27 mars).

${ }^{25}$ Aujourd'hui 25 mars.

${ }^{26}$ Sud-Ouest 24 mars.

${ }^{27}$ Libération 20 mars. À noter aussi que le fils de J. Rottner est lui-même urgentiste en pédiatrie à Mulhouse.

${ }^{28}$ Le Point 17 avril.

${ }^{29}$ Le Monde 27 mars.

${ }^{30}$ Sud Ouest 24 mars.

${ }^{31}$ AFP 16 mars, Aujourdhui 25 mars.

${ }^{32}$ Les Echos 19 mars. 
${ }^{33}$ Par exemple BFM 21 mars.

${ }^{34}$ Aujourd'hui 16 mars.

${ }^{35}$ BFM 21 mars.

${ }^{36}$ Une trentaine d'articles au total abordent son action ou le citent.

${ }^{37}$ Articles conclusifs du Point 8 et 10 avril, et du Monde, 10 avril.

${ }^{38}$ Sud Ouest et Les Echos, $1^{\text {er }}$ avril ; Le Figaro, 2 avril.

${ }^{39}$ AFP 2 avril. J. Rottner sur twitter le 2 avril. L'est républicain le 4.

${ }^{40}$ L'Est Républicain le 5 avril, Bien public et AFP le 6 avril, Le Point 8 avril.

${ }^{41}$ Le Figaro 2 avril

${ }^{42}$ RTL, cité par Le Figaro 2 avril.

${ }^{43}$ Le Point, 8 avril

${ }^{44}$ Très grand nombre d'articles. Voir par exemple l'enquête en 5 épisodes dans Le monde (4 au 7 mai) des deux journalistes d'investigation Davet et Lhomme consacrés par leur best seller d'entretiens officieux avec le président Hollande.

${ }^{45}$ L'Est Républicain 12, 17 et 20 avril, AFP 15 avril, Le monde 20 et 22 avril, Sud Ouest 21 avril, Quotidien du médecin 21 avril, La Tribune 22 avril.

${ }^{46}$ Figaro 24 avril.

${ }^{47}$ Le monde du 22 avril. Les actions citées sont « appel aux dons de masques, achat de masques, rémunération des soignants, attribution de logements et solution de transport toujours pour les soignants, mise en place d'un fonds de résistance et maintenant les tests ».

${ }^{48}$ Le Monde, 27 mars.

${ }^{49}$ Le 23 avril précisément. Voir AFP 23 avril, Le Figaro, Le Monde, L’Est Républicain du 24 avril.

${ }^{50}$ L'avis du CS date du 20 avril. Ces indicateurs sont notamment au niveau régional et départemental, la disponibilité de tests virologiques, la circulation du virus, et le nombre d'hospitalisations.

${ }^{51}$ L'Express 7 mai. Les autres incontournables sont Jean-François Delfraissy, Didier Raoult, Karine Lacombe et Michel Cymes. Consultée le 11 mai, la base Europresse indique plus de 48 références en 3 mois pour Philippe Juvin pour la presse généraliste nationale, contre 46 pour Jean Rottner (et davantage de portraits).

${ }^{52}$ Le Figaro 27 avril.

${ }^{53}$ Le Figaro 8 avril.

${ }^{54}$ Le Figaro 22 avril.

${ }^{55}$ La Tribune 2 avril.

${ }^{56}$ Guerre des masques : Le Point 8 avril (voir aussi Sud Ouest 11 avril). Le 10 avril, 'Coronavirus : les agences régionales de santé, boulets bureaucratiques ?' Le 17 avril : « Cette crise va laisser du monde sur le carreau » (entretien) et le 16 avril «Qu'a fait l'État depuis trois mois ? ». Voir aussi L'Express 16 avril, Le Figaro 16 avril. Ainsi que Valeurs actuelles 20 avril ou Le Figaro 20 avril.

${ }^{57}$ « Ne comptez pas sur eux pour se contenter de parler des TER. Les présidents de région sont partout depuis le début de la crise du coronavirus : dans la presse locale ou nationale, à la télévision ou à la radio, ils multiplient les annonces et accumulent les chiffres, à en faire tourner la tête ». L'Express 16 avril.

${ }^{58} \mathrm{La}$ Gazette des communes, 21 avril.

${ }^{59}$ Le Point 10 avril.

${ }^{60}$ Le Point 17 avril. Cité dans Valeurs actuelles 20 avril. On ne tient pas compte ici de la position évidemment très différente des responsables de l'ARS, notamment de son directeur général Christophe Lannelongue, renvoyé par le gouvernement au cœur de la crise.

${ }^{61}$ Aujourd'hui, 29 avril

${ }^{62}$ Le compte-rendu de la séance n'est pas disponible, mais les propos sont rapportés dans Le Journal du Grand Paris, 30 avril et AFP, 30 avril. Voir aussi l'Est républicain du même jour où est narré « comment la Région a monté sa cellule de crise », et commence par le constat de «l'incapacité de l'État a assurer sa mission de protection » au cœur du tsunami. 